Marijan Bobinac*

Universität Zagreb
UDK 792(497.521.2):81"17/18"

DOI: 10.4312/linguistica.60.2.313-323

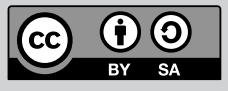

\title{
„EIN GROSSER, HERZERHEBENDER MOMENT“ - ZU DEN WECHSELWIRKUNGEN ZWISCHEN DEM ZAGREBER DEUTSCHEN THEATER UND DER NEUBEGRÜNDETEN KROATISCHEN NATIONALBÜHNE
}

Die Einflüsse aus dem deutschsprachigen Raum intensivieren sich in Kroatien - und darüber hinaus in der Region - seit der Mitte des 18. Jahrhunderts. Damals begannen sich, wie Arnold Suppan bemerkt, deutsch(österreichische) Handwerker, Kaufleute, Beamte und Militärs ,in den Städten des Donau-Drau-Save-Bereiches“ anzusiedeln, welche ,unterstützt von der Merkantilpolitik des absolutistischen Habsburgerstaates [...] einerseits wesentliche Initiativen zur wirtschaftlichen und kulturellen Erneuerung setzten, andererseits den militär-strategischen Aufbau einer organisierten Türkenabwehr in Nordkroatien und Slawonien leisteten“ (Suppan 2008: 639). Aus dieser Zuwanderung entstand in den Städten wie Zagreb/Agram und Osijek/Essek, deren Bevölkerungsstruktur traditionell polyethnisch war, allmählich eine neue, ,kleine bis mittelgroße deutschsprachige Oberschichte“ (Suppan 2008: 690), die z. B. in Zagreb fünf Prozent der Einwohnerzahl selten übertraf. ${ }^{1}$ Von dieser Bevölkerungsgruppe sind vielfältige Initiativen in allen Sphären des gesellschaftlichen Lebens, so auch der Kultur hervorgegangen, ihre Anwesenheit trug wesentlich auch zur Entstehung eines kulturell heterogenen Umfelds bei, in dem das Deutsche, eine zu diesem Zeitpunkt bereits entwickelte Kultursprache, eine dominante Stellung eingenommen hatte.

Unter den Beispielen aus der Kultur- und Mediensphäre lassen sich insbesondere öffentliche Theateraufführungen herausstreichen, die in Zagreb auf Deutsch spätestens seit 1749 gegeben wurden. Zunächst ging es allerdings um ,mit Ausschluß der Öffentlichkeit veranstalteten Vorstellungen“, die von dilettierenden Adeligen gegeben wurden. „Für die Geschichte des deutschen Theaters in Zagreb“ - wie Blanka Breyer, die als erste diesen Gegenstand gründlich erforscht hat - sollen diese Vorstellungen allerdings ,,von keiner Bedeutung“2 (Breyer 1938: 10) gewesen sein. Den Anfang der öffentlichen Wirkung der deutschen Theaterkunst, vermittelt durch reisende deutschsprachige Schauspielertruppen, datiert Breyer mit dem Jahre 1780, fügt allerdings hinzu, dass über dieses und einige darauffolgende Jahre keine Informationen zum Repertoire eruiert werden konnten (Breyer 1938: 11). Für den Zeitraum nach 1784 und die nachfolgenden Jahrzehnte, genauer bis 1860, als die deutschsprachigen Vorstellungen in

mbobinac@ffzg.hr

1 Vgl. z. B. statistische Angaben zur deutschsprachigen Bevölkerung in Zagreb (wie auch anderen Städten der Monarchie) am Ende des 19. und Anfang des 20. Jahrhunderts in: Suppan (2008: 183).

2 Breyer hat das Repertoire des deutschen Theaters im Zeitraum 1780-1840 erforscht. Die Angaben über das Repertoire dieser Bühne zwischen 1840 und 1860 vgl. Batušić 1968. 
Zagreb eingestellt wurden, konnten die Spielpläne fast lückenlos rekonstruiert werden, wobei zwischen der Periode bis 1840, in der in Zagreb nur auf Deutsch gespielt wurde, und der Periode von 1840 bis 1860, als neben Deutsch gelegentlich auch auf Kroatisch gespielt wurde, zu unterscheiden ist.

Gattungsmäßig entsprach das Repertoire der Zagreber deutschen Bühne durchaus jenem in anderen vergleichbaren Städten Mittel- und Osteuropas: Es reichte von der klassizistischen Tragödie Schillers über das populäre Lustspiel Kotzebues und Ifflands bis zu den neuesten Erfolgen aus den Wiener Vorstädten, wobei Bäuerle, Raimund, Nestroy und Friedrich Kaiser am häufigsten vertreten waren. Deutschsprachige Aufführungen wurden in Zagreb vor allem von „Adeligen, deutschen Offizieren, fremden Händlern und höheren Beamten besucht", aber auch „ein Großteil des Zagreber Bürgertums jener Zeit [habe] zweifellos Deutsch verstanden" (Deželić 1901: 6) und daher zum potenziellen Publikum deutschsprachiger Vorstellungen zugehört. Gespielt wurde an mehreren Orten in der Stadt, vor allem in einigen Aristokratenpalais, bevor 1834 das erste öffentliche Theaterhaus erbaut wurde und als Spielstätte bis 1895 diente (dem Jahre, in dem der prächtige Helmer-Fellner-Bau, der heute noch das Kroatische Nationaltheater beherbergt, eröffnet wurde).

Ein Jahrhundert lang also - von der Mitte des 18. bis zur Mitte des 19. Jahrhunderts - wurde das Theaterleben in Zagreb von deutschsprachigen Truppen geprägt, deren Auftritte eine dort zu jener Zeit seltene Möglichkeit zu Unterhaltung und Belehrung boten. Die lang andauernde Dominanz der deutschsprachigen Bühnenkunst - wie auch der deutschsprachigen Kultur überhaupt - in Zagreb begann sich im zweiten Drittel des 19. Jahrhunderts, mit dem Durchbruch des Illyrismus, der kroatischen nationalen Integrationsbewegung, immer mehr als problematisch herauszustellen: Auf der einen Seite sahen die Illyristen im fremdsprachigen Theater ein großes Hindernis für die Entwicklung der nationalen Kultur, andererseits waren ihnen die Erfahrungen der routinierten deutschsprachigen Truppen von größter Bedeutung für die Gründung einer Nationalbühne, eines Institutes, in dem sie ein wichtiges Mittel für die Transponierung ihrer Ideen erblickten.

Der Gründung einer neuen nationalen Kultur, und in diesem Zusammenhang auch eines Nationaltheaters, musste allerdings die Schaffung einer neuen kroatischen Standardsprache vorausgehen, da die bis dahin benutzten Idiome den Herausforderungen einer neuen Zeit nicht gewachsen waren. Nachdem man um 1830 die kajkawische Mundart, die in Nordkroatien in der öffentlichen und literarischen Verwendung üblich war, verworfen und die štokawische zum Standard erkoren hatte, konnte man ein Jahrzehnt später auch mit den Vorstellungen in der neuen, zunächst als illyrisch bezeichneten Sprache beginnen. Diese Aufgabe fiel der sogenannten Vaterländischen Schauspielgesellschaft (Domorodno teatralno društvo) zu, die in Zagreb 1840 und 1841 wirkte. Die Tätigkeit dieses Ensembles markiert nicht nur das Aufkommen der modernen kroatischen Schaubühne, sondern zugleich auch den Anfang des Prozesses, der zum Niedergang des deutschsprachigen Theaters in Zagreb 1860 führte. ${ }^{3}$

3 Zur Geschichte des kroatischen Dramas und Theaters im 19. Jahrhundert vgl. Batušić (1976, 1978, 1986); Cindrić (1969). Die Angaben zum Spielplan kroatischer Theater vgl. Hećimović (1990). Zur Einstellung deutscher Vorstellungen in Zagreb vgl. Car (2002). 
Es wurde schon darauf hingewiesen, wie ambivalent sich die Illyristen der deutschsprachigen Schauspielkunst gegenüber verhielten. Die Erkenntnis, dass die älteren kroatischen Theatertraditionen, vor allem die Aufführungen des Zagreber Priesterseminars in der kajkawischen Mundart, keinen fruchtbaren Boden für die Umsetzung ihrer Anliegen boten, zwang sie schließlich dazu, nach der Logistik sowie nach dramaturgischen und theaterpraktischen Kenntnissen der deutschsprachigen Truppen zu greifen.

Noch bevor es 1840 zu einer engeren Zusammenarbeit zwischen den kroatischen Theaterenthusiasten und den deutschsprachigen Schauspielergruppen gekommen ist, haben einige deutsche Theaterprinzipale in ihren Vorstellungen auf die nationale Begeisterung in Zagreb reagiert. So hat Joseph Schweigert, der Leiter der Zagreber Schauspielertruppe in den 1830er Jahren, ein Theaterstück unter dem Titel Das Schwarze Kreuz auf Medvedgrad (1835) geschrieben, das die lokale, oft als „vaterländisch“ verstandene Thematik in die geläufige Dramaturgie der Wiener Volkstheatertexte integriert.

Unter den Zagreber Prinzipalen ragt insbesondere der Name Heinrich Börnsteins hervor, der die deutsche Theatertruppe in den Jahren 1839 und 1840 leitete und während seiner Tätigkeit auch zum Durchbruch der ersten Vorstellungen im neuen kroatischen sprachlichen Standard verhalf. ${ }^{4}$ In diesem Zusammenhang verfasste er auch eine der ersten dramaturgischen Schriften der neueren kroatischen Schaubühne unter dem Titel Bey der Begründung einer illyrischen National-Bühne, welche - ins Kroatische von Ljudevit Gaj, einem der führenden Vertreter der illyristischen Bewegung, übersetzt - in der Zeitschrift Danica ilirska im November 1839 veröffentlicht wurde. Bei seinem Aufenthalt in Zagreb hat sich Börnstein auch als Herausgeber der 1839 begründeten deutschsprachigen Kulturzeitschrift Croatia betätigt und in diesem Organ die kulturpolitischen Bemühungen der Illyristen mit großen Sympathien verfolgt. So hat er die erste Vorstellung der Vaterländischen Schauspielgesellschaft 1840 in seiner Zeitschrift folgendermaßen begrüßt:

Es ist ein großer, herzerhebender Moment, wenn eine Nation zum ersten Male, ihre geliebte National-Sprache [...] öffentlich in's Leben treten sieht, und so war es auch hier. Der Enthusiasmus des übervollen Hauses ist nicht zu beschreiben, und mit nationeller Begeisterung wurden alle jene Stellen aufgenommen, die sich auf die Würde der Nationalität und auf die angestammte Treue des Croaten an seinen König und sein Vaterland bezogen. ${ }^{5}$

Der Enthusiasmus des festlichen Anlasses mischt sich hier unverkennbar mit der paternalistischen Attitüde des höher entwickelten, zivilisierten „Herrn“, der seinem „Untertan“ großmütig bei der Gründung einer nationalen Institution hilft und diese Tat sogleich für das offizielle Habsburger Narrativ der „Vielheit in der Einheit“ in

\footnotetext{
4 Gespielt wurde das Geschichtsdrama Sofija i Juran ili Turci kod Siska von Ivan Kukuljević Sakcinski am 10. Juni 1840.

5 Croatia, 01.-03.07.1840.
} 
Anspruch nimmt. Hinzuzufügen wäre allerdings, dass Börnstein in der Einführung ,illyrischer" Vorstellungen auch an eigene geschäftliche Vorteile dachte. ${ }^{6}$

Als Höhepunkt der Zusammenarbeit zwischen der deutschen Truppe und den kroatischen Theateraktivisten kann eine merkwürdige Vorstellung angesehen werden, die unter Mitwirkung beider Ensembles im August 1841 stattgefunden hat: Bei dieser Gelegenheit wurde nämlich Theodor Körners historisches Trauerspiel Zriny (UA 1812), das die türkische Belagerung der südungarischen Festung Sigeth 1566 und deren heldenhafte Verteidigung durch die ungarisch-kroatische Truppe des Titelhelden zum Thema hat, zweisprachig auf die Bühne gebracht - die als Kroaten dargestellten christlichen Helden des Dramas sprachen Kroatisch, bzw. Illyrisch, die türkischen sprachen hingegen Deutsch. Über diese Inszenierung hat etwas ausführlicher nur die Zeitschrift Croatia berichtet und dabei insbesondere die zweisprachige Konzeption der Vorstellung in den Mittelpunkt gerückt:

\begin{abstract}
Der Gedanke, ein Stück in zwei verschiedenen Sprachen aufzuführen, scheint im ersten Augenblicke ein wenig barock, doch in diesem Falle der Grundbedingung des Drama's, dem Wahren näher zu liegen, als wenn dieses in einer Sprache gegeben worden wäre. Zwei Völker ringen in ungleichem Kampfe für ungleiche Güter: Moslemim auf einer Seite, auf der anderen Croaten; gehetzte Sklavenwuth in der ersten, edler Freiheitsmuth in der zweiten Fronte; die eine Parthei repräsentiert der welthistorische Soliman, die andere der durch seinen individuellen Heroismus größere Zrinyi. Daß der edle Croatenheld in seiner Muttersprache redet, ist natürlich; in welchem Idiom soll aber der ,Beherrscher des Orients' auf einer deutschen Bühne sprechen? - Ganz natürlich türkisch, wenn er wahr erscheinen soll. Da aber das Türkische deutschen Mimen doch zu spanisch klingt, so mußte man, auch wieder natürlich, beim Deutschen bleiben, der Originalsprache des Körner'schen Drama's. So also sprachen in diesem „Zriny“ die Croaten ilirisch, die Türken deutsch. Ich glaube nicht, daß die Illusion durch dieses Compositum mixtum eine besondere Störung erlitten. ${ }^{7}$
\end{abstract}

Dass die Theaterillusion einer solchen Produktion nicht gestört wurde und die Vorstellung im damaligen Zagreb normal gespielt werden konnte, hängt natürlich mit dem Bilingualismus des Theaterpublikums zusammen, einem Phänomen übrigens, dem auch die jahrzehntelange Anwesenheit des deutschsprachigen Theaters in vielen mittel- und osteuropäischen Städten, so auch in der kroatischen Hauptstadt zu verdanken war. Im Falle der zweisprachigen Zriny-Vorstellung kam dieser Tatbestand offenbar auch dem Problem der Glaubwürdigkeit des Bühnengeschehens entgegen, dessen sich der Rezensent bewusst ist: Die beiden gegnerischen Seiten haben ja in der historischen

6 Dass deutsche Schauspieler im Zeitraum zwischen 1840 und 1860, in denen auf der Zagreber Bühne in zwei Sprachen gespielt wurde, in einer weitaus günstigeren materiellen Lage als ihre kroatischen Kollegen waren, steht jedoch auf einem anderen Blatt. Vgl. dazu: Breyer, Batušić (1968) und Cindrić.

7 Croatia, 27.08.1841. 
Realität tatsächlich unterschiedliche Sprachen gesprochen. Wenn man auch annimmt, dass Zriny und seine Umgebung Kroatisch gesprochen haben, so kann es sicherlich nicht stimmen, dass sich die Türken miteinander auf Deutsch unterhielten. Damit ist natürlich auch etwas anderes verbunden, die Möglichkeit nämlich, dass man die Deutschösterreicher im national berauschten Kroatien der 1840er Jahre wegen ihrer dominanten Position in der Monarchie mit den Türken, die in den südslawischen Kulturen traditionell als Erzfeinde betrachtet wurden, verwechseln könnte. Beim Lesen der Besprechung in der Croatia kann man auch ein leises diesbezügliches Unbehagen des Rezensenten verspüren, welches er allerdings geschickt zu umgehen und ins Humoristische zu überleiten weiß.

Der Konflikt zwischen der deutschsprachigen und kroatischen Kultur, der sich hier auf der symbolischen Ebene klar abzeichnet, wird sich allerdings in den 1850er Jahren in vielerlei Hinsicht verschärfen und schließlich zur Einstellung deutschsprachiger Vorstellungen in Zagreb 1860 führen. Am Anfang der 1840er Jahre, als mit gemeinsamen Kräften Körners Zriny auf die Bühne gebracht wurde, reichten die Kräfte der vaterländischen Schauspielgesellschaft für eine so aufwendige Aufführung noch immer nicht aus, so dass nicht nur deutsche Schauspieler die Rollen der Türken auf Deutsch spielten, sondern auch ihr leitender Vertreter Vincenz Schmidt die Rolle des christlichen Titelhelden Zriny auf Kroatisch mit großem Erfolg gab, obwohl er das einheimische Idiom überhaupt nicht beherrschte.

Aufschlussreich scheint im Zusammenhang der Zriny-Aufführung auch eine andere Beobachtung zu sein. In der Rezension der Croatia ist nämlich vom Spätromantiker Theodor Körner und dessen Stück, das lange zum festen Bestandteil des deutschen Literaturkanons gehörte, heute aber als ästhetisch belanglos angesehen wird, wenig die Rede; herauslesen lässt sich aber, dass der Autor und sein Werk ein hohes Ansehen in der zeitgenössischen Öffentlichkeit genossen. Neben hoher ästhetischer Wertschätzung wurde diesem Bühnenwerk im 19. Jahrhundert in mehreren nationalen Kontexten auch eine überaus starke politische Relevanz zuteil: Körner, der nach einer blitzartigen Karriere als Wiener Hoftheaterdichter 22-jährig in den ersten Monaten der antinapoleonischen Erhebung 1813 als Freiwilliger fiel, verband das Schicksal der christlichen Helden seines Dramas mit der verzweifelten Lage der zeitgenössischen Deutschen, im türkischen Heer suchte er hingegen ein Bild der verhassten französischen Besatzungsmacht, in der Figur des Sultans Soliman einen teuflischen Napoleon darzustellen. Körners desperater Aktionismus aus der Zeit der Befreiungskriege ließ sich auch in den darauffolgenden Jahrzehnten im Zusammenhang mit dem schwierigen Zustandekommen des deutschen Nationalstaates für verschiedene kulturpolitische Zwecke verwerten (vgl. Bobinac 2008: 55-94).

Ungeachtet dieser deutschnationalen Deutung konnte sich die Geschichte vom kaisertreuen Feldherrn, der durch seinen heroischen, obwohl aussichtslosen Widerstand das riesige Türkenheer am geplanten Zug auf Wien hindert, problemlos auch ins patriotisch-dynastische Narrativ der Donaumonarchie einfügen. In der älteren kroatischen und ungarischen Literatur hatte der Zriny-Stoff allerdings schon lange davor eine zentrale Stellung, die im 19. Jahrhundert im Rahmen der national-integrativen Bewegungen nicht 
nur aufrechterhalten wurde, sondern auch die Ausmaße eines nationalen Mythos erhielt: So wurde Nikolaus von Zriny in Ungarn zu einem magyarischen, in Kroatien zu einem kroatischen bzw. slawischen Leonidas stilisiert - und in diesem Sinne ist er auch in einige andere slawische nationale Narrative innerhalb der Donaumonarchie eingegangen. ${ }^{8}$

Mit hoher Bühnenwirksamkeit und mehrfacher kulturpolitischer Relevanz haben zweifellos auch die Initiatoren der Zagreber Zriny-Inszenierung 1841 gerechnet. Das merkwürdige, aus heutiger Sicht eher multikulturell anmutende Theaterereignis, das sich inmitten heftiger Debatten über die politische und kulturelle Emanzipation der Kroaten abspielte, verweist auf ein verwirrendes Nebeneinander unterschiedlicher Kräfte, dessen Komplexität von später entstandenen national-romantischen Narrativen in vielerlei Hinsicht vereinfacht bzw. beseitigt wurde. Gerade der Aufstieg des modernen kroatischen Nationaltheaters und der Niedergang der Zagreber deutschsprachigen Bühne im zweiten Drittel des 19. Jahrhunderts stellen ein solches, für österreichisch-ungarische Verhältnisse charakteristisches Spannungsfeld dar, welches nicht nur auf unterschiedliche Entwicklungsgrade einzelner nationaler Kulturen, sondern gleichzeitig auch auf deren vielfältige Überschneidungen, Berührungen und Gemeinsamkeiten hinweist.

Die hohe Komplexität dieses Phänomens lässt sich nur schwer aus einer einzigen Forschungsperspektive darstellen, so dass für dessen differenziertere Analysen sich eher eine Kombination von mehreren, vor allem kulturwissenschaftlich geprägten Forschungsansätzen - auch wenn sie unterschiedliche Ausgangspositionen haben - empfiehlt. ${ }^{9}$ So wird man - wenn wir bei unserem Beispiel bleiben - in der Existenz vieler deutschsprachiger Theater außerhalb der administrativ und kulturell dominanten deutschsprachigen Zentralgebiete der Donaumonarchie sehr wohl auch einen deutlichen Hinweis auf das Gefälle zwischen Zentrum und Peripherie festhalten können. In einer solchen kulturellen Asymmetrie innerhalb des Habsburger Reichs wäre dann für kulturwissenschaftlich inspirierte Forscher insbesondere das Zusammenspiel zwischen - wie Wolfgang Müller-Funk in einem anderen Kontext vorschlägt - „Superioritätsgefühle[n] [...] auf der einen“ sowie „Ansprüche[n] auf Emanzipation auf der anderen Seite“ (Müller-Funk 2002: 22) vom Interesse.

Die Existenz einer weit verbreiteten deutschsprachigen Theaterlandschaft könnte man andererseits - in Anlehnung an eine These von Moritz Csáky - auch als eine wesentliche Erscheinungsform jener ,übergeordnete[n], verbindende[n], kulturelle[n] Codes“ betrachten, die sich „,in Zentraleuropa aufgrund kontinuierlicher Diffusionsprozesse" (Csáky 2002: 44) ausgebildet haben. Indem man also nicht nur an einer eigenen „Nationalkultur", sondern auch an Elementen anderer, ,fremder" Kulturen partizipierte, kam es dazu, ,,daß Mehrfachidentitäten begünstigt wurden und Multipolarität die individuellen und kollektiven Identitäten bestimmte“, was sich auch „,in der praktischen

8 Von dieser parallelen Entwicklung zeugt auch die ungefähr gleichzeitige Drucklegung von Körners Zriny in ungarischer (1826), tschechischer (1838) und kroatischer (1840) Übersetzung, aber auch das Erscheinen einer ungarischen Ausgabe in französischer Sprache (1835). Näher über die nationalen Zuweisungen in den einzelnen Zriny-Übersetzungen vgl. in Bobinac (2008: 62-67).

9 Vgl. z. B. die Beiträge von Wolfgang Müller-Funk, Moritz Csáky, Clemens Ruthner und John Neubauer in: Müller-Funk u. a. (2002). 
Zwei- und Mehrsprachigkeit vieler Bewohner“ (Csáky 2002: 45) reflektierte. So nimmt es auch kein Wunder, dass in Städten wie Zagreb, die trotz einer eindeutigen Mehrheit der einheimischen Bevölkerung stark vom deutschsprachigen Element geprägt waren, die deutsche Bühnenkunst lange florieren konnte.

Einer solchen, für multinationale Staatsgebilde charakteristischen kulturellen Hybridität wirkten bekanntlich die Vertreter der national-integrativen Bewegungen bei den kleineren Völkern der Donaumonarchie mit ihrer Forderung entgegen, eine ,eigene' kollektive nationale Identität zu konstruieren - zunächst in der Sprache, bald darauf auch in kulturellen Teilsystemen wie Theater. Die Bestrebungen nationaler Aktivisten richteten sich insbesondere gegen die dominierende deutschsprachige Kultur, wobei sie - nur auf den ersten Blick paradox - gerade von der spezifisch deutschen Auffassung von einer organischen nationalen Kultur ausgingen. Wenig änderte sich dabei an der Tatsache, dass die deutschsprachige Kultur des Habsburger Reiches sich nicht national-aggressiv wie jene reichsdeutsche gebärdete, sondern sich eher auf dynastisch-patriotische und zentralstaatlich-restaurative Werte fokussierte und dabei - trotz all ihrer hegemonialen Züge - auch das Emanzipationsstreben peripherer Völker, wie man dies an der Zagreber Zriny-Inszenierung sieht, respektierte.

Der symbolischen Entgegensetzung des deutschen und kroatischen Elements im Zagreber Theater am Anfang der 1840er Jahre folgte - wie anfangs erwähnt - ein echter Konflikt in der historischen Realität zwei Jahrzehnte später. Als im Frühjahr 1860 eine neue Truppe engagiert wurde, sah es vorerst so aus, als ob die schon seit Jahren praktizierte Zweisprachigkeit der Zagreber Bühne auch in Zukunft fortgesetzt werden würde. Ein halbes Jahr später, im November 1860, wurde jedoch die deutschsprachige Vorstellung eines Stückes von Charlotte Birch-Pfeiffer durch laute Rufe kroatischer Patrioten unterbrochen. Nach diesem Ereignis, das die kroatische Theaterpublizistik später auch als „Vertreibung deutscher Schauspieler“ zu bezeichnen pflegte, mussten fast siebzig Jahre vergehen, bis im kroatischen Nationaltheater wieder eine deutschsprachige Vorstellung - es war ein Gastspiel des Wiener Burgtheaters 1928 - stattfinden konnte (vgl. Car 2002).

Die Einstellung deutschsprachiger Vorstellungen in Zagreb, obwohl sie von einem späteren Betrachtungswinkel aus überraschend und abrupt aussehen könnte, ging logisch aus den großen politischen und kulturellen Veränderungen hervor, die mit dem Revolutionsjahr 1848 und der daraufhin folgenden Periode des Neoabsolutismus in Österreich angetreten sind. Nachdem sich nämlich die patriotische Begeisterung durch das Scheitern der Revolution und die Aufhebung der konstitutionellen Ordnung verflüchtigt hat, wurde in den 1850er Jahren die Arbeit an der Gründung einer ständigen nationalen Schaubühne fortgesetzt, eine Arbeit, die trotz zahlreichen Hindernissen politischer, sozialer oder finanzieller Natur langsam vorankam und am Ende des Jahrzehnts erste künstlerische Erfolge brachte. Im Gegensatz dazu konnten die deutschen Schauspieltruppen - trotz gelegentlicher Erfolge - ihr früheres Prestige nicht mehr wahren, so dass ihre Vorstellungen am Ende der 1850er Jahre immer weniger besucht wurden.

Die spürbare Liberalisierung aller gesellschaftlichen Bereiche der Monarchie, die im Zusammenhang mit der Wiedereinführung des Konstitutionalismus nach dem 
verlorenen Krieg in Italien 1859 stand, ermöglichte auch einen freieren Diskurs über die prekäre Lage des Zagreber Theaters. Neue Akzente setzte auf diesem Gebiet vor allem die neubegründete Tageszeitung Pozor, die im Gegensatz zu den beiden offiziösen Blättern Narodne novine und Agramer Zeitung einen oppositionellen Kurs einschlug: „Dem deutschen Theater gegenüber sind wir völlig gleichgültig, aber so viel können wir doch sagen, dass wir es für kein Unglück halten, wenn die deutschen Aufführungen auch weiterhin niemand besucht." ${ }^{\prime 10}$

Dieser höchst polemische Ton könnte zur Annahme verleiten, dass über die Zagreber Theaterkrise vom Herbst 1860 eine heftige Pressedebatte geführt wurde. Doch das Gegenteil war der Fall: Wenn man sich nur auf die Berichterstattung und Kommentare der damaligen Printmedien stützen würde, so ließe sich die plötzliche Einstellung deutscher Vorstellungen kaum nachvollziehen. Weder Pozor noch eine andere Zeitung hat sich eindeutig für ein Verbot deutscher Vorstellungen in Zagreb ausgesprochen; als sie aber tatsächlich eingestellt wurden, hat dagegen niemand öffentlich protestiert. Dass den Debatten über diesen Vorfall auch die noch immer scharfen Zensurbestimmungen im Wege standen, liegt auf der Hand. Und trotzdem ist es verwunderlich, dass dieser, in den späteren Zeiten als historisch bezeichnete Augenblick in der zeitgenössischen Presse kaum erwähnt, ja fast verschwiegen wurde.

Bei der Rekonstruktion der Ereignisse vom 24. November 1860 berufen sich die Theaterhistoriker vor allem auf die Erinnerungen einiger Zeugen aus dem Theatermilieu. Problematisch erscheint dabei aber die Tatsache, dass sie viel später, um die Jahrhundertwende, veröffentlicht wurden, in einer Zeit also, in der das kroatische Theater schon organisatorisch und artistisch profiliert war und der öffentliche Diskurs sich viel freier gestalten konnte. Aus diesen Schriften, die aus der Feder von Theaterpublizisten Nikola Andrić und Milan Ogrizović stammen, kann man erfahren, dass während der letzten deutschsprachigen Vorstellung einige Zuschauer zu pfeifen begannen, worauf sich das ganze Auditorium - mit Ausnahme weniger Deutschen - der Demonstration anschloss. Da die Darstellung, die sowieso auf Erinnerungen an weit zurückliegende Ereignisse beruht, von den beiden Verfassern noch zusätzlich dramatisiert und fiktionalisiert wurden, lässt sich als Tatsache nur festhalten, was man ohnehin weiß - dass nämlich nach heftigen Protesten des Publikums deutsche Vorstellungen in Zagreb eingestellt wurden.

Nach Ogrizović sollen noch am selben Abend Dimitrija Demeter und Josip Freudenreich, die beiden führenden kroatischen Dramatiker und Theateraktivisten jener Zeit, die Einführung des ausschließlich kroatischen Spielbetriebs beschlossen haben; obwohl diese Entscheidung sofort in Praxis umgesetzt wurde, fehlen auch von ihr jegliche schriftlichen Spuren. Auch im Gesetz über das kroatische Nationaltheater, das vom kroatischen Landtag ein Jahr danach, 1861, verabschiedet wurde, ist von einem Verbot deutschsprachiger Vorstellungen keine Rede. Und immerhin wurde dieses Verbot zu einem „ungeschriebenen Gesetz", an dem in den folgenden Jahrzehnten nie gerüttelt wurde.

Nach der enthusiastischen Stimmung im November 1860, die sich bald verflüchtigte, folgte eine harte, ernüchternde Arbeit an der Konstituierung des kroatischen Ensembles

10 Pozor, 10.10 .1860$. 
und Spielplans. Einen Anschluss an die europäischen Entwicklungstendenzen fand das kroatische Nationaltheater erst in den 1890er Jahren; zugleich war das auch die Zeit, in der die nationale Integration der Kroaten schon ihren Höhepunkt erreichte und unter den Intellektuellen auch Diskussionen über lange verschwiegene Themen eröffnet wurden so auch jene über die Einstellung deutschsprachiger Vorstellungen im Zagreber Theater. Es ist nicht zu übersehen, dass darin auch das national-romantische Narrativ, welches vor allem das „Fremde“, das „Unerwünschte“ aus dem angeblich „reinen“ Eigenen auszuschließen sucht, einen seiner ersten Höhepunkte erreicht (vgl. Deželić 1901).

\section{Literatur}

ANDRIĆ, Nikola (1895) Spomen knjiga hrvatskog zemaljskog kazališta pri otvaranju nove kazališne zgrade. Zagreb: Tiskarski zavod „Narodnih novina“.

BARIC, Daniel (2013) Langue allemande, identité croate. Au fondement d'un particularisme culturel. Paris: Armand Colin.

BATUŠIĆ, Nikola (1968) Uloga njemačkog kazališta u Zagrebu u hrvatskom kulturnom životu od 1840. do 1860, Rad JAZU, Nr. 353, Zagreb: JAZU.

BATUŠIĆ, Nikola (1976) Hrvatska drama od Demetra do Šenoe. Zagreb: Nakladni zavod Matice hrvatske.

BATUŠIĆ, Nikola (1978) Povijest hrvatskog kazališta. Zagreb: Školska knjiga.

BATUŠIĆ, Nikola (Hrsg.) (1986) Hrvatska drama 19. stoljeća. Split: Logos.

BOBINAC, Marijan (2008) Zwischen Übernahme und Ablehnung. Aufsätze zur Rezeption deutschsprachiger Dramatiker im kroatischen Theater. Wrocław - Dresden: ATUT - Neisse Verlag.

BOBINAC, Marijan (2010) „L'élément allemand sux origines du théâtre croate moderne." In: D. Baric et al. (Hrsg.), Mémoire et histoire en Europe centrale et orientale, Rennes: Presses universitaires de Rennes, 189-196.

BOBINAC, Marijan (2014) „Cultural Transfer in the Habsburg Empire. Croatia and German-Language Culture from a Postcolonial Perspective.“ In: D. Göttsche/A. Dunker (Hrsg.), (Post-) Colonialism across Europe. Transcultural History and National Memory, Bielefeld: Aisthesis, 305-319.

BÖRNSTEIN, Heinrich (1839) „O utemeljenju ilirskoga narodnoga kazališta.“ Danica ilirska, 5/46, 181-182.

BREYER, Blanka (1938) Das deutsche Theater in Zagreb 1780-1840. Mit besonderer Berücksichtigung des dramatischen Repertoires. Zagreb: Diss. Universität Zagreb.

CAR, Milka (2002) „Der 24. November 1860 im kroatischen Theater. Die ,Vertreibung' der deutschen Schauspieler.“ Zagreber Germanistische Beiträge, 11, 97-118.

CINDRIĆ, Pavao (Hrsg.) (1969) Hrvatsko narodno kazalište 1894-1969. Enciklopedijsko izdanje. Zagreb: Jugoslavenski leksikografski zavod.

CSÁKY, Moritz (2002) „'Was man Nation und Rasse heißt, sind Ergebnisse und keine Ursachen'. Zur Konstruktion kollektiver Identitäten in Zentraleuropa.“ In: W. Müller-Funk u. a. (Hrsg.), Kakanien revisited. Das eigene und das Fremde (in) der österreichisch-ungarischen Monarchie. Tübingen/Basel: A. Francke, 33-49. 
FRUK, Marina (1997) „Die Illyristen als Mitarbeiter der Zeitschrift ,Croatia“ (18391842).“ Zagreber Germanistische Beiträge, 6, 185-197.

DEŽELIĆ, Velimir (1901) Iz njemačkoga Zagreba. Prinos kulturnoj povjesti [!] Hrvata. Zagreb: A. Scholz.

HEĆIMOVIĆ, Branko (Hrsg.) (1990) Repertoar hrvatskih kazališta. 1840-1860-1980. Zagreb: Globus.

MÜLLER-FUNK, Wolfgang u. a. (Hrsg.) (2002) Kakanien revisited. Das eigene und das Fremde (in) der österreichisch-ungarischen Monarchie. Tübingen/Basel: A. Francke.

OGRIZOVIĆ, Milan (1910) Pedeset godina hrvatskoga kazališta, Zagreb: Uprava Kr. hrv. zem. kazališta.

SUPPAN, Arnold (2008) „'Germans' in the Habsburg Empire. Language, Imperial Ideology, National Identity and Assimilation.“ In: Ch. W. Ingrao/F. A. J. Szabo (eds), The Germans and the East. West Lafayette: Purdue UP, 147-190.

RUMPLER, Helmut (1997) Österreichische Geschichte 1804-1914. Eine Chance für Mitteleuropa. Wien: Ueberreuter.

STANČIĆ, Mirjana (2013) Verschüttete Literatur: die deutschsprachige Dichtung auf dem Gebiet des ehemaligen Jugoslawien von 1800 bis 1945. Wien: Böhlau.

\author{
Zusammenfassung \\ „EIN GROSSER, HERZERHEBENDER MOMENT“ - \\ ZU DEN WECHSELWIRKUNGEN ZWISCHEN DEM ZAGREBER \\ DEUTSCHEN THEATER UND DER NEUBEGRÜNDETEN \\ KROATISCHEN NATIONALBÜHNE
}

In Zagreb - wie auch in anderen vergleichbaren mittel- und osteuropäischen Städten - wurde das Theaterleben lange von Vorstellungen deutschsprachiger Theatertruppen bestimmt. Die frühesten Aufführungen in deutscher Sprache - es ging um geschlossene Vorstellungen einheimischer Aristokraten - gehen auf das Jahr 1749 zurück. Öffentlich wurde in Zagreb auf Deutsch zwischen 1780 und 1860 gespielt, in der Regel ging es um Auftritte deutschsprachiger Schauspielertruppen, die sich für eine oder mehrere Saisonen in der Stadt niedergelassen hatten. - In diesem Aufsatz werden zunächst einige Höhepunkte der deutschsprachigen Bühnenkunst in Zagreb herausgestrichen, daraufhin das ansonsten sehr ambivalente Verhältnis der deutschsprachigen Truppen zu den kroatischen Theateraktivisten vorgeführt, die im zweiten Drittel des 19. Jahrhunderts eine nationale Schaubühne zu organisieren suchten, ein Anliegen, welches schließlich auch zur Einstellung deutschsprachiger Vorstellungen 1860 führte.

Schlüsselwörter: deutschsprachiges Theater in Zagreb, Gründung des kroatischen Nationaltheaters, Kulturtransfer 


\section{Abstract \\ "A GREAT, AN UPLIFTING MOMENT" - ON THE INTERACTIONS BETWEEN THE GERMAN ZAGREB THEATRE AND THE NEWLY FOUNDED CROATIAN NATIONAL THEATRE}

Theatrical life in Zagreb - as well as in other comparable Central and East European cities - had long been dominated by performances of German-speaking theatre companies. The earliest performances in German, staged exclusively for domestic aristocrats, date back to 1749. Public theatrical events were held in German between 1780 and 1860 , usually performed by German-speaking troupes, which settled in the city for one or more seasons. Presenting initially some highlights of the German-speaking stage art in Zagreb, the paper then focuses on the often ambivalent relationship between the German-speaking troupes and Croatian theatre activists in the second third of the $19^{\text {th }}$ century. Seeking to establish a new national theatre during this period, the activists eventually succeeded in 'expelling' German actors from the Zagreb theatre and introducing an exclusively Croatian repertoire in 1860.

Keywords: German-speaking theatre in Zagreb, establishing of the Croatian National theatre, cultural transfer

\section{Povzetek}

„VELIK, SRCE DVIGAJOČ TRENUTEK“ - VZAJEMNI VPLIVI MED

ZAGREBŠKIM NEMŠKIM GLEDALIŠČEM IN NOVOUSTANOVLJENIM HRVAŠKIM NARODNIM GLEDALIŠČEM

V Zagrebu - in tudi drugih primerljivih srednje- in vzhodnoevropskih mestih - so gledališko delovanje dolgo določale predstave nemško govorečih gledaliških skupin. Najzgodnejše izvedbe v nemškem jeziku - šlo je za predstave nemških aristokratov, zaprte za javnost - segajo v leto 1749. Javne predstave so se v nemščini izvajale med 1780 in 1860, praviloma pa je šlo za nastope nemških gledaliških skupin, ki so prišle v mesto za eno sezono ali več sezon. - V članku so najprej izpostavljeni nekateri vrhunci nemške gledališke umetnosti, nato pa je opisan sicer zelo ambivalenten odnos nemško govorečih gledaliških skupin do hrvaških gledaliških aktivistov. Ti so v drugi tretjini 19. stoletja začeli organizirati hrvaški gledališki oder, kar je tudi dokončno privedlo do ustavitve nemških predstav leta 1860 .

Ključne besede: nemško govoreče gledališče v Zagrebu, ustanovitev hrvaškega narodnega gledališča, kulturni transfer 